\title{
Effect of Massage Therapy on Physiologic Responses in Patients with Congestive Heart Failure
}

\author{
Safieh Jamali ${ }^{1}$, Somayeh Ramezanli², Marzieh Kargar Jahromi ${ }^{3}$, \\ Azam Zare ${ }^{4}$ and Farzad Poorgholami ${ }^{5 *}$ \\ ${ }^{1}$ Research Center for Social Determinants of Health, \\ Jahrom University of Medical Sciences, Jahrom, Iran. \\ ${ }^{2}$ Medical,Surgical Nursing, Faculty Member, Jahrom University of Medical Science, Jahrom, Iran \\ ${ }^{3}$ Community Health Nursing, Faculty Member, Jahrom University of Medical Science, Jahrom, Iran \\ ${ }^{4}$ Medical, Surgical Nursing, Alzahra Heart Charity Hospital, \\ Shiraz University of Medical Sciences, Shiraz. Iran. \\ ${ }^{5}$ Medical, Surgical Nursing, Faculty Member, Jahrom University of Medical Science, Jahrom, Iran
}

http://dx.doi.org/10.13005/bbra/2044

(Received: 15 January 2016; accepted: 01 March 2016)

\begin{abstract}
Congestive heart failure ( $\mathrm{CHF}$ ) is the inability of the myocardium to pump enough blood to meet the body's metabolic demands. This study was conducted to assess the effects of effect of massage therapy on physiologic responses in patients with congestive heart failure. This study was a quasi-experimental, done with the permission of Jahrom University of Medical Sciences that conducted on two CCU wards of hospitals of Jahrom. Fifty participants were recruited to participate in this study within 24 hours after admission to the cardiac ward. The massages were begun 3 days after hospitalization in order not to disturb their medical treatment plan. Blood pressure, heart rate, respiratory rate, and blood oxygen saturation data were collected 30 minutes before and after back massage. A total of 50 individuals participated in this study. After 3 days of massage, participants' systolic BP $(p<0.01)$, diastolic BP $(p<0.01)$, HR $(p<0.01)$, and respiratory rates $(p<0.01)$ were significantly decreased. Also, the participants' oxygen saturation levels showed significant improvement $(p<0.01)$. Based on the findings of the present study, massage therapy was effective in blood pressure, heart rate, respiration rate and $\mathrm{O} 2$ saturation in patients with CHF. Therefore we suggest that massage therapy be used as a complementary method to stabilize their vital signs.
\end{abstract}

Keyword: Massage Therapy, Physiologic Responses, Congestive Heart Failure, Patients

Congestive heart failure (CHF) is the inability of the myocardium to pump enough blood to meet the body's metabolic demands (AHA, 2011). The most common causes of CHF are hypertension and coronary artery disease (Schub et al, 2011).

CHF is characterized by several complex symptoms that are difficult to control and result in a high prevalence of re-hospitalization, morbidity,

\footnotetext{
* To whom all correspondence should be addressed. Tel.: 98-71-5434-1501;

E-mail:FarzadPoorgholami1393@gmail.com
}

and mortality across the world. Common physical symptoms of CHF are tachycardia, shortness of breath, decrease in arterial blood oxygenation (Pao2), and discomfort (Davies et al, 2000).

Almost $50 \%$ of patients with CHF experience stress symptoms (Huffman et al, 2008). Patients with CHF feel uncertain, anxious, and depressed (Chen et al, 2010) Negative emotions (i.e., anxiety and depression) appear to be more common in patients with CHF than in healthy individuals (Moser, 2000). Elderly patients with heart failure had higher levels of anxiety than the healthy elderly. Moreover, it increases the length 
of hospital stay and decreases the quality of life (Moser et al, 2010).

Different methods have been applied to reduce anxiety and its consequences. Therapeutic touch and massage are of the oldest anxiolytic methods (Sherman et al, 2005). Massage is a systematic form of touch that manipulates soft tissues of the body to promote comfort and healing (Harris, 2010).

Back massage appeared to reduce diastolic BP, respiration, perceived psychological distress, and pain in the preparatory time for cardiac catheterization (McNamara et al, 2003). However, a randomized clinical study demonstrated that 30minute massage therapy sessions after cardiac surgery did not improve physical condition (e.g., heart rate and BP). (Albert et al, 2009). While there have been multiple studies examining the effects of massage on the treatment of various medical conditions, there has been no research done to investigate whether massage improves physiologic response in patients with CHF in Iran. Aim

The aims of this study were to investigate effect of massage therapy on physiologic responses in patients with congestive heart failure in Iran.

\section{METHODSANDMATERIALS}

\section{Setting}

This study was a quasi-experimental, done with the permission of Jahrom University of Medical Sciences that conducted on two CCU wards of hospitals of Jahrom. Subjects were recruited using convenience sampling.

\section{Data collection}

The data collection tool included two parts. The first part consisted of questions about demographic data.The second part was a sheet for recording the physiologic indicator (Blood pressure, heart rate, respiratory rate, and blood oxygen saturation).

Blood pressures were measured from the left arm, using a mercury sphygmomanometer, after resting for 15 minutes lying on back. The patients' heart rate and $\mathrm{O} 2$ saturation were monitored on the patients monitor. Respiration rate was also measured for one minute without patient's notice. During massage therapy, the patients' heart rhythm was also being monitored.

\section{Inclusion criteria}

The criteria for inclusion: The participants were diagnosed with NYHA Class I-IV heart failure by a cardiac specialist, being literate, being conscious, and a long of stay more than 3 days, and willing to participate in the study.

\section{Exclusion criteria}

The participants with potential confounders, such as a thrombosis greater than 1 cm by the Doppler echography, back-pressure ulcers, acute pulmonary edema, respiratory failure, or cardiac tamponed, a cardiac arrest in the past 72 hours, severe dyspnea, a cardiac pacemaker, a pulse rate less than $60 / \mathrm{min}$, a dialysis fistula and a history of bone fracture in the past 2 months and a history of receiving massage therapy were excluded.

\section{Procedure}

This study was approved by the Research Council and the Research Ethics Committee of Jahrom University of Medical Sciences. The researchers also obtained permission from the hospital authorities and an informed consent was obtained before data collection. The standard massage procedure was guided by an assistant professor of physical therapy.

Fifty participants were recruited to participate in this study within 24 hours after admission to the cardiac ward. The massages were begun 3 days after hospitalization in order not to disturb their medical treatment plan.

Protocols for massage varied in previous studies (Jane et al, 2008). The intensity and duration of massage ranged from one session of 3-45 minutes to 15 sessions over 4 weeks (Jane et al, 2009; Post-White et al, 2003). To be consistent with the research aim to improve physical indicators, the protocol of massage for each participant in the intervention group, one session of massage therapy (for about 60 minutes) was done. For massage facilitation almond oil was used to massage back, shoulder deltoid muscles, front part of legs, arms, forearms, back of thighs, palms and fingers, metatarsus, front part of thighs, back of feet and toes, belly and chest, auxiliaries and neck muscles of the patient. Massage techniques used included static massage, surface tension techniques, stretching massage, superficial lymph unload, and transverse friction techniques (Shiri, 2010). 
Massage has been done for 3 days consecutively. Massage was arranged one hour before a meal or two hours after a meal. Before the massage, each participant was asked to sit up and position himself/herself on a bedside table with a pillow to cushion. Curtains were drawn to ensure the participants' privacy. Blood pressure, heart rate, respiratory rate, and blood oxygen saturation data were collected 30 minutes before and after back massage.

\section{Data analysis}

The massage effects on HR, respiratory rate, $\mathrm{BP}$, and $\mathrm{O} 2$ saturation were examined across time with the paired t-test, separately, comparing each value of the post intervention measures with the value of pre-intervention measures. A repeatedmeasures analysis of variance was used to capture the effects of patterns on outcome measures.

\section{RESULTS}

A total of 50 individuals participated in this study. The proportion of male and female participants was 41.35.The mean age of the total cases was 69.13 (SD=11.82) years. Totally, $89.16 \%$ of the patients were married, $68.33 \%$ were employed and the others were retired or unemployed. The education of most of patients (58.33\%) was at the level of primary school and the rest was at the level of secondary school or higher education. None of the patients had a history of massage therapy .With regard to the particular features of their conditions, the largest group (45.72) had been diagnosed with CHF less than 6 months earlier; most participants (70.31\%) were NYHA class III (marked limitation of physical activity, only comfortable at rest).(Table 1 ).

Table 1. Demographic Data of Research Participants

\begin{tabular}{llc}
\hline Characteristic & & Value \\
\hline Mean age (years) & & $69.13(\mathrm{SD}=11.82)$ \\
Sex [\% (women/men)] & & $41 / 35$ \\
Level of education & Elementary & 58.33 \\
& Secondary or higher & 41.67 \\
Marital status & Married & 89.16 \\
& Single or widowed & 10.84 \\
Employment & Employed & 68.33 \\
& Retired or Unemployed & 31.67 \\
CHF Diagnose & less than 6 months & 45.72 \\
& 6 months or more & 54.28 \\
CHF classes & I & 6.33 \\
& II & 23.36 \\
& III & 70.31 \\
\hline
\end{tabular}

Table 2. Changes in Physiologic Indicators after Intervention $(n=50)$

\begin{tabular}{lccccc}
\hline $\begin{array}{l}\text { Time } \\
\text { Variable }\end{array}$ & $\begin{array}{c}\text { Pre intervention } \\
\text { Mean } \pm \text { SD }\end{array}$ & $\begin{array}{c}\text { Day 1 } \\
\text { Mean } \pm \text { SD }\end{array}$ & $\begin{array}{c}\text { Day 2 } \\
\text { Mean } \pm \text { SD }\end{array}$ & $\begin{array}{c}\text { Day3 } \\
\text { Mean } \pm \text { SD }\end{array}$ & F $^{\mathrm{a}}$ \\
\hline Systolic BP (mm Hg) & $136.35(18.85)$ & $129.53(19.19)$ & $126.23(16.65)$ & $123.85(16.98)$ & $18.82^{*}$ \\
Diastolic BP (mm Hg) & $81.95(10.66)$ & $77.72(10.60)$ & $75.26(9.87)$ & $72.81(9.54)$ & $13.25^{*}$ \\
Heart rates (times/min) & $95.12(16.16)$ & $91.04(13.66)$ & $87.98(12.34)$ & $85.16(12.19)$ & $25.18^{*}$ \\
Respiration (times/min) & $23.56(4.18)$ & $21.03(3.48)$ & $21.73(3.52)$ & $20.03(3.05)$ & $5.57^{*}$ \\
Oxygen saturation (\%) & $93.62(3.32)$ & $93.99(2.94)$ & $94.74(3.33)$ & $95.76(3.62)$ & $41.92^{*}$
\end{tabular}

${ }^{*} \mathrm{p}<0.01$

$\mathrm{aF}, \mathrm{F}$ value analyzed by using repeated measures. SD, standard deviation; and $\mathrm{BP}$, blood pressure. 
As shown in Table 2, After 3 days of massage, participants' systolic BP ( $<<0.01)$, diastolic BP $(\mathrm{p}<0.01), \operatorname{HR}(\mathrm{p}<0.01)$, and respiratory rates $(\mathrm{p}<0.01)$ were significantly decreased. Also, the participants' oxygen saturation levels showed significant improvement $(\mathrm{p}<0.01)$.

\section{DISCUSSION}

Results of the present study showed that massage therapy have beneficial effects on physiologic response of patients hospitalized in CCU by balancing systolic and diastolic blood pressures, heart rate and respiration rate. Some investigators found no significant effects of massage therapy on psychological responses (Cowen et al, 2006; Combron et al, 2006). However, findings of the present study are consistent with the studies conducted by Haji Hoseini et al., (2006) and Shaban et al. (2004). This is consistent with the results Hernandez-Reif et al. 2000 In parallel with the results of systolic BP, the massage group also showed significant chronic reduction in diastolic BP after two, three and four days of massage therapy and acute significant reduction of $7 \mathrm{mmHg}$ after session 1 .

Massage may induce a sense of comfort and relaxation and then endorphins may be secreted (Tamparo et al, 2011), vessels would be dilated, the blood flow increases within the superficial vessels of body (Eungpinichpong, 2008) and blood pressure will be reduced.

The recent study showed that the cardiopulmonary distress declined significantly after the first day of massage, and this happened again after the second and third days of massage.

In the literature review, it was found that of all the physiologic indicators of the effect of massage, HR was the strongest indicator of relaxation.

The reduction of heart rate after the massage may also be related to the anxiolytic and Para sympathomimetic effects of massage (Buttagat et al, 2011; Lindgren et al, 2010).

This is supported by Diego and Field who showed that the massage applied at medium pressure for 15minutes caused increase in the highfrequency component of HR variability which reflected an increase in vagal activities. In addition, there was a decrease in the ratio of low-frequency component to high-frequency component of HR variability which indicates a change from sympathetic activities to parasympathetic activities (Diego, 2009).

Breathing rates also declined significantly. After the massages, respiratory rates showed significant decline on day 1 and day 3, but not on day 2. Further study would be needed to verify the relationship between changes in respiratory rate and frequency of massage.

Labyak and Metzger, after using met analysis on massage patients, found that the patients' breathing frequency declined by $6.4 \%$ after the massages (Labyak, 1997).

After participating in massage for 2 days, participants' blood oxygen saturation levels had significantly increased compared to preintervention in this study. Massage can also improve pulmonary function and promote circulation when the amount of blood in the pulmonary artery increases. The increased amount of blood has more opportunity to exchange gases, enabling more oxygen supply throughout the body and raising the oxygen saturation level (Field, 2002).

The observed alterations in the vital signs and can be important because these changes would reduce the myocardial oxygen demand. It can be recommended that massage therapy be used to balance the vital signs of patients admitted in CCU.

Overall, this study demonstrated the feasibility of implementing a back massage program, and the acceptability and perceptions of benefit in patients with CHF.

\section{CONCLUSION}

Based on the findings of the present study, massage therapy was effective in blood pressure, heart rate, respiration rate and $\mathrm{O} 2$ saturation in patients with CHF. Therefore we suggest that massage therapy be used as a complementary method to stabilize their vital signs. However, further research can be suggested with daily repeating of massage therapy, evaluation vital signs at more times after the massage therapy sessions.

\section{Ethical aspects}

The study was approved by the institutional Ethics Committee of the University 
Hospital, jahrom, Iran. Prior to this study, patients were informed of the purpose of the research. Participants were informed of their right

to refuse to participate in or to withdraw from the study at any stage. Anonymity and confidentiality of participants were maintained.

\section{Conflict of Interest}

The authors declare that there is no conflict of interests regarding the publication of this paper.

\section{ACKNOWLEDGMENTS}

Hereby, the respected personnel and patients at Hospital of Jahrom who have cooperated in gathering material are acknowledged.

\section{REFERENCES}

1. Albert NM, Gillinov AM, Lytle BW, et al. A randomized trial of massage therapy after heart surgery. Heart Lung 2009; 38: 480-490.

2. American Heart Association. Heart failure. Online document at: www.heart.org/Conditions/ Heart Failure Accessed September 27, 2011.

3. Buttagat V, Eungpinichpong W, Chatchawan U, Kharmwan S. The immediate effects of traditional Thai massage on heart rate variability and stress related parameters in patients with back pain associated with myofascial trigger points. Journal of Bodyw Mov Ther 2011; 15: 15-23.

4. Chen HM, Clark AP, Tai LM, Lin CC. Selfreported health related quality of life and sleep disturbances in Taiwanese people with heart failure. J Cardiovasc Nurs 2010; 25: 503-513.

5. Chen W, Liu G, Yeh S, Chiang M, Fu M, Hsieh Y. Effect of back massage intervention on anxiety, comfort, and physiologic responses in patients with congestive heart failure. J Alterna Complement Med 2013; 19: 464-70.

6. Combron JA, Dexheimer J, Coe P. Changes in blood pressure after various forms of therapeutic massage: A preliminary study. J Altern Complement Med 2006; 12: 65-70

7. Cowen VS, Burkett L, Bredimus J, Evans DR, Lamey S, Neuhauser T, et al. A comparative study of Thai massage and Swedish massage relative to physiological and psychological measures. J Bodywork Movement Ther 2006; 10: $266-75$

8. Davies N, Curtis M. providing palliative care in end-stage failure. Prof Nurs 2000; 15: 389-392.
9. Diego MA, Field T.Moderate pressure massage elicits a parasympathetic nervous system response. International Journal of Neuroscience. 2009; 119(5): 630-638.

10. Eungpinichpong W. Therapeutic Thai Massage. Bangkok: Chonromdek Publishing House. 2008.

11. Field T. Massage therapy. Med Clin North Am 2002; 86: 163-171.

12. Haji Hoseini F, Avazeh A, Elahi N, Shariati A, Sori $\mathrm{H}$. The effect of massage on comatos patients' vital signs, hospitalized in intensive care units. J Arak Univ Med Sci 2006; 3: 1-10.

13. Harris M, Richards KC. The physiological and psychological effects of slow-stroke back massage and hand massage on relaxation in older people. J Clin Nurs 2010; 19: 917-926.

14. Hernandez-Reif M, Field T, Krasnegor J, Hossain Z ,Theakst H, Burman I, "High blood pressure and associated symptoms were reduced by massage therapy," Journal of Bodywork and MovementTherapies, 2000; 4(1): 31-38.

15. Huffman JC, Smith FA, Blais MA, Januzzi JL, Fricchione GL. Anxiety, independent of depressive symptoms, is associated with inhospital cardiac complications after acute myocardial infarction. J Psychosom Res 2008; 65: 557-563.

16. Jane SW, Wilkie DJ, Gallucci BB, et al. Systematic review of massage intervention for adult patients with cancer. Cancer Nurs 2008; 31: E24-E35.

17. Jane SW, Wilkie DJ, Gallucci BB, et al. Effects of a full-body massage on pain intensity, anxiety, and physiological relaxation in Taiwanese patients with metastatic bone pain: A pilot Study. J Pain Symptom Manage 2009; 37: 754 763.

18. Labyak DE, Metzger BL. The effects of backrub on the physiological components of relaxation: A meta-analysis. Nurs Res 1997; 46: 59-62.

19. Kargar Jahromi M, Javadpour SH, Taheri L, Poorgholami F. Effect of Nurse-Led Telephone Follow ups (Tele-Nursing) on Depression, Anxiety and Stress in Hemodialysis Patients. Global Journal of Health Science. 2016; 8(3): 168-173.

20. Lindgren L, Rundgren S, Winsö O, Lehtipalo S, Wiklund U, Karlsson M, et al. Physiological responses to touch massage in healthy volunteers. Auton neurosci 2010; 158, 105110.McNamara ME, Burnham DC, Smith C, Carroll DL. The effects of back massage before diagnostic cardiac catheterization. Altern Ther Health Med 2003; 9: 50-57.

21. Moser DK, Worster PL. Effect of psychosocial factors on physiologic outcomes in patients with 
CHF. J Cardiovasc Nurs 2000; 14: 106-115.

21. Moser DK, Dracup K, Evangelista LS, et al. Comparison of prevalence of symptoms of depression, anxiety, and hostility in elderly patients with heart failure, myocardial infarction, and a coronary artery bypass graft. Heart Lung 2010; 39: 378-385.

22. Poorgholami F, Abdollahifard F, Zamani M, KargarJahromi M, BadiyepeymaJahromi Z. The Effect of Stress Management Training on Hope in Hemodialysis Patients. Global Journal of Health Science. 2016; 8(7). 165-171

23. Poorgholami F, Mansoori P, Montaseri Z, Najafi K. Effect of Self-Care Education with and without Telephone Follow-Up on the Level of Hope in Hemodialysis Patients; A Randomized Clinical Trial. IJCBNM. 2016; 4(2): 90-97.

24. Sabetgadam M, Poorgholami F, Badiyepeymaie Jahromi F, Parandavar F, Kalani N, Rahmanian E. Effect of Self-Care Education by Face-toFace Method on the Quality of Life in Hemodialysis Patients. Global Journal of Health Science. 2016; 8(6):140-147.

25. Poorgholami F, Rahmanian Koshkaki A, Kargar Jahromi M, Parniyan R. A Study of the Influence of Group-Based Learning of Stress Management on Psychology Symptoms Levels of Hemodialysis Patients. Global Journal of Health Science. 2016; 8(11): 62-68.

26. Post-White J, Kinney ME, Savik K, et al. Therapeutic massage and healing touch improves symptoms in cancer. Integr Cancer Ther 2003; 2: 332-344.

27. Schub T, Cabrera G. Heart failure and atrial fibrillation. Online document at: http:// web.ebscohost.com/nrc/ detail? Accessed April 4, 2011.

28. Shaban M, Haji Amiry P, Mehran A, Kahrary S. Evaluation of immediate effect of foot massage on patient's vital signs in a general intensive care unit. Hayat 2004; 10: 71-80.

29. Sherman KJ, Cherkin DC, Kahn J, Erro J, Hrbek A, Deyo RA, et al. A survey of training and practice patterns of massage therapists in two US states. BMC complement altern med 2005; 14: 13.

30. Shiri M. Massage therapy (2nd ed.). Tehran: Aeeizh. 2010.

31. Tamparo CD, Lewis MA. Diseases of the Human Body (Diseases of the Human Body (5th ed.). Philadelphia: F.A. Davis Co. 2011. 